\title{
Identification of Multi-Faults in GNSS Signals using RSIVIA under Dual Constellation*
}

\author{
Shuchen Liu ${ }^{a b}$, Jan-Jöran Gehrt ${ }^{a c}$, Dirk Abel ${ }^{a d}$, \\ and René Zweigel ${ }^{a e}$
}

\begin{abstract}
This publication presents the development of integrity monitoring and fault detection and exclusion (FDE) of pseudorange measurements, which are used to aid a tightly-coupled navigation filter. This filter is based on an inertial measurement unit (IMU) and is aided by signals of the global navigation satellite system (GNSS). Particularly, the GNSS signals include global positioning system (GPS) and Galileo. By using GNSS signals, navigation systems suffer from signal interferences resulting in large pseudorange errors. Further, a higher number of satellites with dual-constellation increases the possibility that satellite observations contain multiple faults. In order to ensure integrity and accuracy of the filter solution, it is crucial to provide sufficient fault-free GNSS measurements for the navigation filter. For this purpose, a new hybrid strategy is applied, combining conventional receiver autonomous integrity monitoring (RAIM) and innovative robust set inversion via interval analysis (RSIVIA). To further improve the performance, as well as the computational efficiency of the algorithm, the estimated velocity and its variance from the navigation filter is used to reduce the size of the RSIVIA initial box. The designed approach is evaluated with recorded data from an extensive real-world measurement campaign, which has been carried out in GATE Berchtesgaden, Germany. In GATE, up to six Galileo satellites in orbit can be simulated. Further, the signals of simulated Galileo satellites can be manipulated to provide faulty GNSS measurements, such that the fault detection and identification (FDI) capability can be validated. The results show that the designed approach is able to identify the generated faulty GNSS observables correctly and improve the accuracy of the navigation solution. Compared with traditional RSIVIA, the designed new approach provides a more timely fault identification and is computationally more efficient.
\end{abstract}

${ }^{*}$ The development of current publication is part of the joint research project GALILEOnautic 2 (grant number 50NA1808), which is supported by the German Federal Ministry for Economic Affairs and Energy. Basis for the support is a decision by the German Bundestag.

${ }^{a}$ Institute of Automatic Control, RWTH Aachen University, Germany

${ }^{b}$ E-mail: s.liu@irt.rwth-aachen.de, ORCID: https://orcid.org/0000-0003-4685-491X

${ }^{c}$ E-mail: j.gehrt@irt.rwth-aachen.de, ORCID: https://orcid.org/0000-0003-4348-2110

${ }^{d}$ E-mail: d.abel@irt.rwth-aachen.de, ORCID: https://orcid.org/0000-0003-0286-3654

${ }^{e}$ E-mail: r.zweigel@irt.rwth-aachen.de, ORCID: https://orcid.org/0000-0003-2440-2138 
Keywords: RSIVIA, RAIM, GNSS, fault detection and identification, Kalman filter

\section{Introduction}

As described in the market report from the European Global Navigation Satellite Systems Agency (GSA) [5], satellite-based navigation will substantially contribute to the future innovation of self-driving vehicles. In autonomous applications, especially in safety-critical scenarios, a false estimation of vehicle states can result in catastrophic accidents. Therefore, a reliable navigation solution with high integrity is required. To maintain the integrity of a satellite-based navigation system, the faulty GNSS observations caused by signal interferences and other possible reasons shall be detected, identified and excluded. Ever since the operation of open service of the newly developed EU satellite navigation system Galileo, the combination of GPS and Galileo provides more available satellites in view for the modern navigation systems. However, a higher number of satellites also increases the possibility that satellite observations contain a fault or even multi-faults. Therefore, identification of multi-faults becomes a crucial and challenging task to maintain the integrity of GNSS-based navigation systems.

The previous work [10] presents the development of a fault detection and exclusion (FDE) algorithm of GNSS measurements. The approach operates as an extension of a tightly-coupled navigation filter, which integrates the measurements from GNSS, an inertial measurement unit (IMU) and a Doppler velocity log (DVL) [6]. In [10], FDE bases on the receiver autonomous integrity monitoring (RAIM) approach with parity space [12], which is a pure statistical method. RAIM predicts pseudorange residuals, which are based on the estimated reference vehicle state using least square method, and uses the residuals to detect and identify pseudorange faults. Since RAIM is a pure statistic method and based on single fault assumption, it might not always be adequate, if multiple measurements are faulty. This can be observed in [11]. This work concentrates on multi-fault identification, when the conventional statistic based approach cannot certainly provide a correct identification solution.

In recent years, an alternative localization method, set inversion via interval analysis (SIVIA), is developed under such concern. SIVIA estimates a trust region of the state space fulfilling a predefined confidence level. The basic operations of interval analysis are introduced in [7] and applied to realize robot localization in [8]. Further, [13] shows an example of integrating velocity information from DVL to compute the guaranteed robot trajectories using interval analysis. With respect of GNSS application, robust SIVIA (RSIVIA) approach is applied for satellite positioning in [3] [4], which allows to estimate the trust region of antenna position with existing erroneous pseudorange measurements. Hereby, it is possible to identify outliers in the GNSS observations by checking the compatibility of each GNSS 
measurement and the estimated trust region. The main drawback of this approach is its computational load, because RSIVIA begins with an initial guess of an arbitrary big box, bisects it into small boxes and operates on them separately and iteratively. In addition, RSIVIA usually runs at a lower rate (1 Hz in [4]) than GNSS observations (10 Hz in current navigation filter), such that the timely fault identification can not be guaranteed. This can result in corrupted state estimation, when there is an increasing pseudorange error [14].

The present publication proposes a FDE scheme to benefit the advantages of RAIM and RSIVIA and compensates the disadvantages of them, which is illustrated in Fig. 1. Once a new set of GNSS measurements is available, fault detection and identification (FDI) of RAIM are carried out iteratively. Eventually, a fault alert is generated from RAIM and used as a trigger for RSIVIA, if RAIM still detects a fault but is not capable to identify it. This reduces the computational load and enables a more timely fault identification. When RSIVIA is triggered for the further FDI task, it is executed in an iterative process, which refers to RSIVIA GNSS update in Fig. 1: it starts with the assumption that no fault exists in the observed measurement space. Whenever an empty trust region is returned, RSIVIA assumes one more fault existing in the measurements. This iterative process continues until a non-empty trust region is estimated. The rest of the faulty measurements are identified by checking the consistency of the measurements with the resulting trust region.

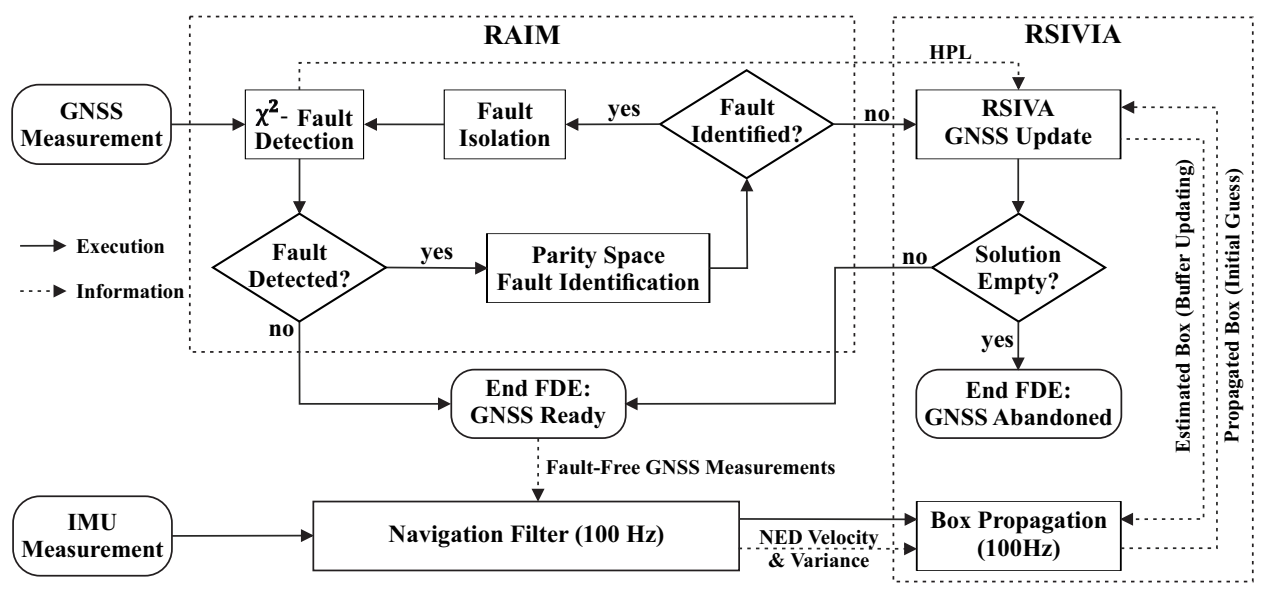

Figure 1: Scheme of GNSS FDE involving RAIM and RSIVIA

To reduce the computational load, RSIVIA is initialized with an arbitrary big box and the first trust region is estimated only with GNSS measurements. After that, the estimated velocity information and its variance from the navigation filter are used to propagate the trust region from last step. In this way, further RSIVIA steps start with the propagated trust box as initial guess, whose size is much smaller than an arbitrary big box. 
Furthermore, output from the conventional RAIM is used to aid RSIVIA. Estimated horizontal protection level (HPL) is used for parameterizing minimal error bound, whose deterministic calculation is not given in [3] [4] for GNSS application.

The designed approach is evaluated in post-processing environment with the recorded data, with respect of correctness of FDI, accuracy improvement of the navigation solution and reduce of computational load. In order to reproduce the test scenario in a post-processing environment, all sensors and GNSS correction data are recorded in real-world tests at Galileo test and development environment (GATE) in Berchtesgaden, Germany. To validate the FDI functionality, the fault identification results are compared with report of generated faulty Galileo measurements from IFEN GmbH.

The paper is structured as follows: First, the state vector of navigation filter and RAIM are introduced briefly. Then, the method of RSIVIA using GNSS measurements is described as the basic. At the end of methodology part, the practical integration of velocity outputs from navigation filter and RAIM is given. In the experimental validation part, the measurement setup and target hardware are described, experimental results are evaluated and discussed afterwards. Finally, the last section draws the conclusion and provides an outlook for future developments of integrity monitoring within inertial navigation system.

\section{Methodology}

\subsection{Navigation Filter}

The basic concepts and equations of a tightly-coupled navigation filter are introduced in [6] [9]. This section concentrates on introducing the filter outputs. In total, 18 states are predicted within a strap-down algorithm using the measurements from $3 \mathrm{D}$ accelerometer and 3D gyroscope,

$$
\boldsymbol{x}=\left[\begin{array}{lllllll}
\boldsymbol{p}_{e b}^{e} & \boldsymbol{v}_{e b}^{n} & \boldsymbol{q}_{b}^{n} & \boldsymbol{b}_{a} & \boldsymbol{b}_{g} & c_{b} & c_{d}
\end{array}\right]^{T} .
$$

The state vector $\boldsymbol{x}$ contains position $\boldsymbol{p}_{e b}^{e}$ of the IMU body-frame origin in EarthCentered-Earth-Fixed $(\mathrm{ECEF})$ coordinates $(3 \times 1)$ and the velocity of body-frame origin $\boldsymbol{v}_{e b}^{n}(3 \times 1)$, which is in navigation frame North-East-Down (NED) coordinates with respect to ECEF frame. Furthermore, a quaternion $\boldsymbol{q}_{b}^{n}$ for alignment of body frame and NED frame $(4 \times 1)$, accelerometer bias $\boldsymbol{b}_{a}(3 \times 1)$ and gyroscope bias $\boldsymbol{b}_{g}(3 \times 1)$ are estimated. Additionally, a tightly coupled system needs to estimate receiver clock bias $c_{b}$ and drift $c_{d}$ for correction of pseudo- and deltaranges. The process and measurement model used for Kalman update is given in [6] [9]. It should be stressed that the velocity information $\boldsymbol{v}_{e b}^{n}$ is given in NED coordinates, which is one important reason for choosing NED coordinates as the operation navigation frame for RSIVIA. The other reason is that using NED coordinates makes it easier to distinguish between horizontal and vertical components. Focusing on horizontal components is important for most autonomous applications. 


\subsection{RAIM with Parity Space}

The previous work [10] presents the development of a FDE extension based on RAIM. The necessary equations of residual-based RAIM for pseudorange fault detection are given in [1] [10]. Further, using parity space based RAIM for fault identification is introduced in [12] [10]. Hereby, only necessary theory is explained, helping to understand the integration of RAIM in Sec. 2.5.

In General, RAIM uses the pseudorange residuals to detect and identify faulty GNSS measurements. Pseudoranges are predicted, based on the estimated reference vehicle states. The pseudorange residuals are calculated as the difference between measured and predicted pseudoranges. According to statistics, with $\nu$ independent standard normal random variables, the sum of their squares satisfies chi-squared distribution with $\nu$ degree of freedom (DOF). Assuming that the pseudorange measurement noise satisfies the white mean Gaussian distribution with various standard deviation. After using pseudorange measurements to estimate the 4 unknowns by using least square approach, the normalized predicted residual of pseudoranges $\nu$ shall satisfy $N-4$ DOF chi-squared distribution. $\mathrm{N}$ is the number of available pasudorange measurements. Otherwise, RAIM shall declare that an error occurs. The fault identification is done iteratively with the help of parity space using Bayes Rule, assuming all satellites having the same prior probability of being faulty [12][10]. It should be noticed that RAIM also estimates HPL, which is a function of pseudorange variances, the geometric satellite constellation and the predefined parameters, i.e. false alarm rate and missed detection probability. The estimated HPL is used in Sec. 2.5 to parametrize the minimal error bound of RSIVIA.

\subsection{RSIVIA with Pseudorange Measurements}

\subsubsection{Interval Analysis Basics}

Interval analysis (also called interval computation) is the operation on intervals instead of algebraic operation on numbers, although the basic operators are the same as in algebraic operation:,,$+- \times, \div, \sin$, tan, exp. The computation of intervals is defined in [7], as follows

$$
[a] \diamond[b]=[\{a \diamond b \in \mathbb{R} \mid a \in[a], b \in[b]\}],
$$

where $[a]$ and $[b]$ are intervals. Further, the high dimensional interval is defined as a box. $\diamond$ can be any of the algebraic operations listed above. By applying interval analysis on satellite-based navigation, the pseudorange measurement equation is expressed as

$\left[\rho_{i}\right]=\sqrt{\left(\left[x_{n, i}^{s}\right]-\left[x_{n}^{a}\right]\right)^{2}+\left(\left[x_{e, i}^{s}\right]-\left[x_{e}^{a}\right]\right)^{2}+\left(\left[x_{d, i}^{s}\right]-\left[x_{d}^{a}\right]\right)^{2}}+\left[c_{b}\right], \quad \forall i \in\{1,2, \ldots, N\}$,

where $\boldsymbol{x}^{s}=\left[x_{n, i}^{s}, x_{e, i}^{s}, x_{d, i}^{s}\right]^{T}$ is the $i^{t h}$ satellite position and $\boldsymbol{x}^{a}=\left[x_{n}^{a}, x_{e}^{a}, x_{d}^{a}, c_{b}\right]^{T}$ is the antenna position and receiver clock bias, both in NED frame. $N$ is the number of available pseudorange measurements. 


\subsubsection{Measurement Bounding}

In the context of satellite pseudorange-based navigation, the measurement vector is $\tilde{\boldsymbol{\rho}}=\left[\tilde{\rho}_{1}, \cdots, \tilde{\rho}_{i}, \cdots, \tilde{\rho}_{N}\right]$. Based on the measured pseudoranges $\tilde{\boldsymbol{\rho}}$, the interval of them $[\boldsymbol{\rho}]$ should be estimated. In [4], an approach is proposed, estimating the lower and upper bound of the measurements, tolerating faulty measurements. Allowing a certain number of faulty measurements to estimate the trust box is defined as $q$-relax, where $q$ is the number of tolerated faulty measurements.

Consider a set of $N$ available measurements with the condition of $q$-relax, it means that at least $N-q$ measurements are required to be fault free. It is assumed that the probability of a measurement being faulty satisfies binomial distribution. The probability of $q$-relax condition satisfied is calculated as

$$
P\left(n_{f f} \geq N-q\right)=\sum_{k=N-q}^{N} P\left(n_{f f}=k\right)=\sum_{k=N-q}^{N} \frac{N !}{k !(N-k) !} p_{f f}^{k}\left(1-p_{f f}\right)^{N-k},
$$

where the confidence level $P\left(n_{f f} \geq N-q\right)$ is predefined. Therefore, $p_{f f}$ can be estimated as the only unknown in Eq. (4), which is the probability of each satellite being faulty free. The probability density function of a measurement noise is known as $f(e)$, which is practically assumed to be a white Gaussian distribution. In this way, for each measurement $p_{f f}$ is calculated as

$$
p_{f f}=P(\rho \in[\widetilde{\rho}+a, \widetilde{\rho}+b])=\int_{a}^{b} f(e) d e .
$$

With $p_{f f}$ estimated from Eq. (4), the lower and upper bound can be calculated by minimizing the width of the interval $[a, b]$.

\subsubsection{The RSIVIA Process with GNSS Measurements}

To estimate the trust box of the state vector $\left[\boldsymbol{x}^{a}\right]$, RSIVIA starts with the feasible initial guess $\left[\boldsymbol{x}_{0}^{a}\right]$, which allows to be arbitrarily big and guarantees the true solution of $\boldsymbol{x}^{a}$ inside it. RSIVIA attempts to reduce the size of the initial guess with a contractor $\mathcal{C}$. A contractor is an operator $\mathbb{R}^{n} \rightarrow \mathbb{R}^{n}$ associated to a constraint (in our case Eq. (3)), which returns a box $\mathcal{C}[\boldsymbol{x}] \subseteq[\boldsymbol{x}]$ without losing any vector consistent with the constraint [13]. If the size of the operated box cannot be further reduced by a contractor, it will be bisected into two small boxes and the contractor operation will be repeated for all small boxes remained. This process ends, until the width of all remained boxes is smaller than a predefined error bound $\epsilon$. The detailed design of the RSIVIA process in GNSS applications is given in [3] [4], which includes forward and backward contractor using constraints given in Eq. (3). This RSIVIA operation is summarized as Line 4 in Alg. 1.

Alg. 1 gives the whole process of a bounding box update, when a new GNSS measurement is available. This process starts with a fault-free assumption $(q=0)$ and attempts to estimate the trust region with an increasing $q$. This operation is summarized as GNSS update. The resulting box $\left[\boldsymbol{x}^{a}\right]$ is applied in Eq. (3) to predict 


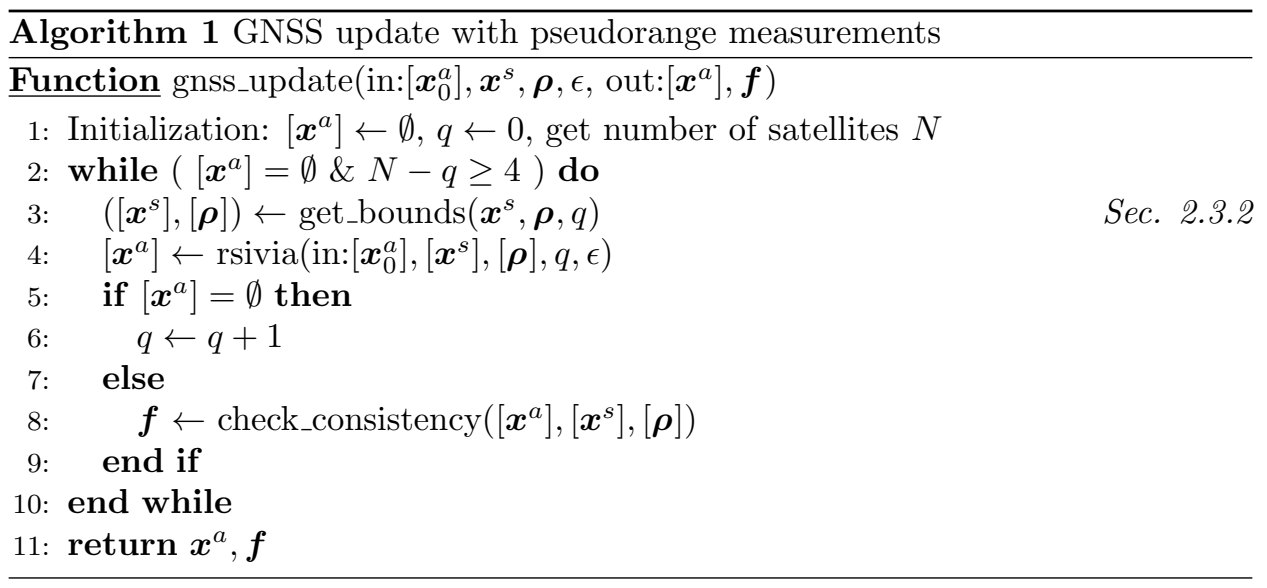

the interval of each pseudorange, with the corresponding box of satellite position $\left[x_{n, i}^{s}, x_{e, i}^{s}, x_{d, i}^{s}\right]$. The faulty measurement is identified when the predicted pseudorange interval has no intersection with the measured one. This consistency check returns a fault vector $\boldsymbol{f}$ consisting of $N$ elements, which are 0 or 1 , representing whether this measurement is fault free.

Still, questions remain in this process, i.e. how should the initial guess $\left[\boldsymbol{x}_{0}^{a}\right]$ and the minimal acceptable error bound $\epsilon$ be chosen, considering both the correctness of fault identification and computational load. These will be answered in Sec. 2.4 and 2.5 , respectively.

\subsection{Integration of Velocity Information in RSIVIA}

In [13], a frame is proposed for guaranteed integration of state equations. An example is given in [13], which uses DVL measurements and differential state constraints to estimate tubes of driven trajectories of an autonomous underwater vehicle (AUV). A tube is defined as an envelope, which encloses an uncertain trajectory. To estimate the tube, a differential tube contractor $\mathcal{C}_{\frac{d}{d}}$ is applied, which consists of a forward contractor $\mathcal{C}_{\frac{d}{d t}}$ and a backward contractor $\mathcal{C}_{\frac{d}{d t}}^{\leftarrow}[13]$.

Due to several reasons, only forward contraction $\mathcal{C}_{\frac{d}{d t}}^{\rightarrow}$ is applied in the current work, using the output velocity information from the navigation filter in Sec. 2.1. First, the propagated box using velocity information is not the output as in [13]. Instead, the propagated box is only used as the initial box $\left[\boldsymbol{x}_{0}^{a}\right]$ for GNSS update in Alg. 1. GNSS update still dominates the result of the trust box estimation. Second, in [13] backward contraction is necessary, because only the DVL velocity measurements are available. Without backward contraction, the size of estimated tubes can never be reduced, because it is the integration of the width of measurement error bound. Due to the usage of GNSS measurements, this is not the situation in current publication. In practice, the estimated velocity is with relative narrow variance, which enables an accurate propagation, without losing integrity 
of the initial guess $\left[\boldsymbol{x}_{0}^{a}\right]$. This will be validated in Sec. 3.2.1. Further, the size of the boxes can be strongly reduced by the GNSS update. Considering the difficulty of operating on multi-rate navigation system with GNSS measurement time delay, only forward contractor with the following differential constraint is applied,

$$
\left[\boldsymbol{x}^{a}\right](t+d t)=\left[\boldsymbol{x}_{0}^{a}\right](t+d t) \cap\left(\left[\boldsymbol{x}^{a}\right](t)+d t \cdot\left[\dot{\boldsymbol{x}}^{a}\right](t)\right) .
$$

As mentioned in Sec. 2.1, all the operations are carried out in NED coordinates, such that $\dot{\boldsymbol{x}}^{a}$ is already given in Eq. (1): $\dot{\boldsymbol{x}}^{a}=\left[\boldsymbol{v}_{e b}^{n} c_{d}\right]^{T}$. The bounding box $\left[\dot{\boldsymbol{x}}^{a}\right]$ is estimated with the output $\dot{\boldsymbol{x}}^{a}$ and its estimated covariance from the navigation filter. The detailed contractor design and discretization are given in [13].

In practice, GNSS measurement is updated with the rate of ca. $10 \mathrm{~Hz}$ and with a time delay of $50 \sim 300$ milliseconds. This time delay is a result of signal processing from the GNSS receiver, after satellite signals are received by the antenna. However, the navigation filter runs at $100 \mathrm{~Hz}$, because a high-rate navigation solution is necessary for autonomous vehicles. Considering the unnegligible delay, a structure is proposed in Fig. 2, which is an example supposing GNSS time delay is 30 milliseconds.

Fig. 2 shows, when no GNSS update is available, the box at $k+1$ step is propagated using $\left[\boldsymbol{x}_{k}^{a}\right]$ and $\left[\dot{\boldsymbol{x}}_{k}^{a}\right]$ from last step. Without additional information of the initial box at $k+1$ step, the initial box $\left[\boldsymbol{x}_{0, k+1}^{a}\right]$ is taken as $[-\mathbf{i n f}$, inf $]$, such that the part after $\cap$ symbol in Eq. 6 dominates the calculation. Once a GNSS update is available, the time of GNSS measurement is estimated by comparing the current time and measured time delay. The propagated box stored in the buffer is located and used as the initial guess $\left[\boldsymbol{x}_{0}^{a}\right]$ for the GNSS update, which is described in Sec. 2.3.3. After GNSS update, all the boxes until the current epoch are propagated again and the corresponding buffer will be replaced.

Fig. 3 shows an one dimensional example, which illustrates the change of estimated upper and lower bound before and after the GNSS update. In this example, the GNSS measurement is received at $k+4$ epoch (current) with time delay of 3 epochs, such that the GNSS update is carried out in the past (at $k+1$ epoch). The bounds at $k+1$ epoch (black) are used as initial guess for GNSS update and narrower bounds (blue) are estimated. The blue bounds are propagated using the stored velocity information in memory until the current time $(k+4$ epoch $)$.

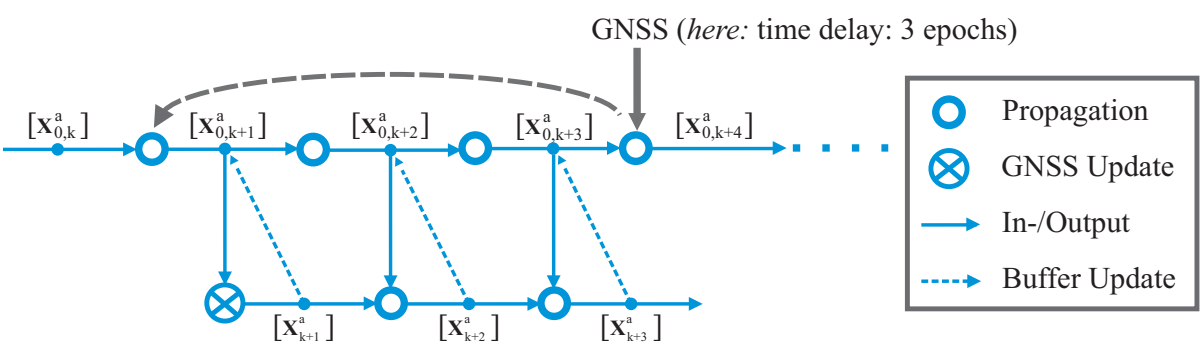

Figure 2: Propagation and GNSS update considering measurement delay 


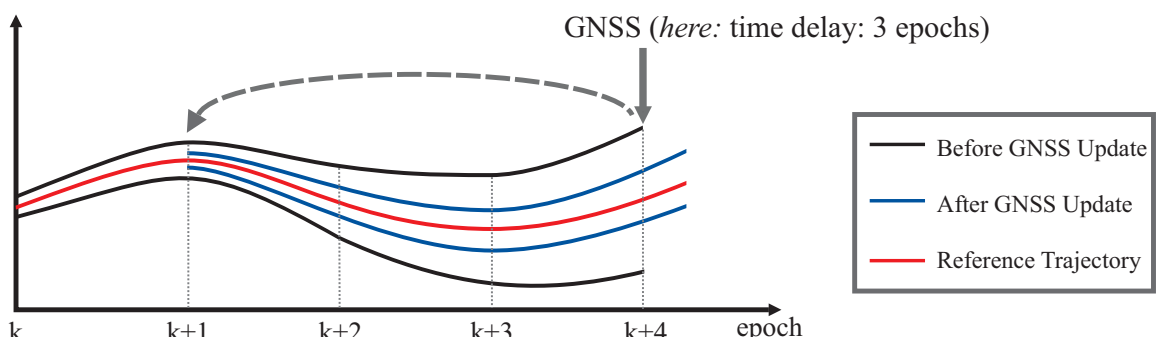

Figure 3: One dimensional (1D) example of integrating velocity information and resulting variance in GNSS update

\subsection{Integration of RAIM}

By integrating velocity information, RSIVIA does not start with an arbitrary box anymore, which reduces the computational load. This can be further improved by triggering the RSIVIA, when a GNSS update is necessary, instead of executing it by each new GNSS measurement. Whether a GNSS update is necessary, is decided by the RAIM fault detection result.

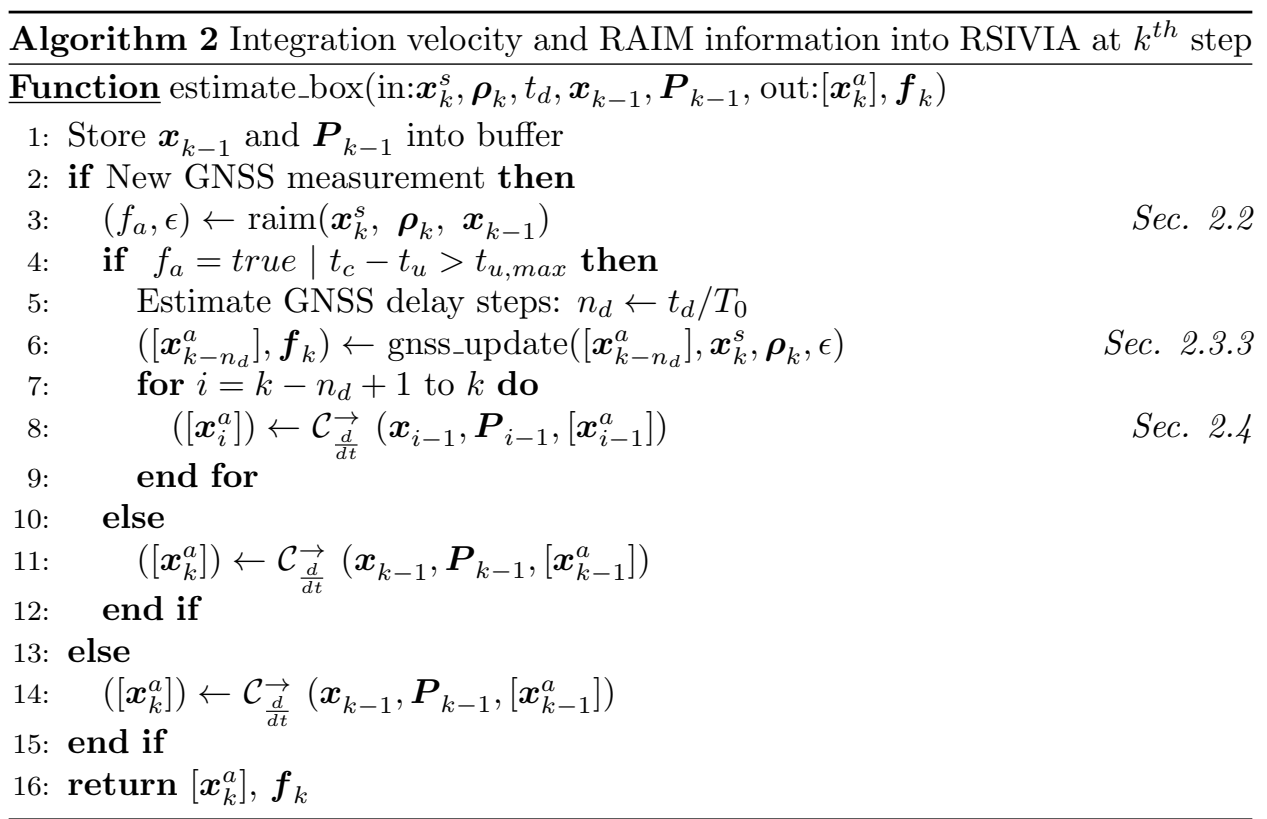

Nevertheless, a situation should be avoided that no GNSS update is executed for a long duration, when no fault is detected by RAIM. Therefore, a parameter $t_{u, \max }$ is introduced, which defines the maximum duration allowed between two 
GNSS updates. The detailed implementation is given in Alg. 2, where $t_{c}$ and $t_{u}$ are the current time and time of last GNSS update, respectively. $t_{d}$ is the GNSS measurement time delay. $T_{o}$ is the navigation filter sample time.

Furthermore, Alg. 2 proposes a new method of parameterizing the minimum error bound $\epsilon$ of RSIVIA, which is a parameter used in Alg. 1. In [8], guaranteed minimum outlier number estimator (GOMNE) is applied, which proposes to reduce the error bound to half of the previous value $(\epsilon \leftarrow \epsilon / 2)$, when the $q$-relax increases. Still, the initial value of $\epsilon$ needed to be parametrized with this method. [2] discusses the choice of the error bound and the pseudorange quality by comparing different settings of $\epsilon$. However, a deterministic calculation of the error bound $\epsilon$ is never given. By introducing the output of RAIM, both satellite constellation and measurement quality are considered (Sec. 2.2).

\section{Experimental Validation}

In this section, the experimental results of the proposed approach for GNSS integrity monitoring as well as pseudorange measurements FDE are given. The results are divided into two parts: first, the evaluation of the dynamic propagation using the velocity information; second, the validation of the FDE capability, the computational load and the accuracy improvement.

\subsection{Measurement Setup and Test Scenario}

The sensor data is recorded on a $900 \mathrm{MHz}$ single core rapid control prototyping (RCP) unit, called MicroAutoBox II from dSPACE. GNSS signals are received and decoded by a Septentrio AstRx3 HDC receiver at a rate of $10 \mathrm{~Hz}$. The communication between the receiver and the RCP unit is achieved via serial interface. For inertial measurement, the setup uses a LORD MicroStrain 3DM-GX4-25 industrialclass 9 DOF IMU-sensor, which is connected via serial interface and provides accelerations, angular rates and magnetometer measurements at a rate of $100 \mathrm{~Hz}$. The receiver provides a pulse per second (PPS). Using the PPS, the communication and processing delays of the receiver are measured (see [8]). In order to reproduce the real-world test scenario in a post-processing environment, all sensors and GNSS correction data are recorded.

Fig. 4 shows the bird eye view of the driven trajectory. The experiment is carried out in the so-called "T-Cross" in Berchtesgaden, Germany, because it is the best test track for the visibility of all three base stations from GATE system. It should be noticed that the driven path in this experiment is in open area. Therefore, it is assumed that, except the generated feared events, the measurements from other satellites are fault free. However, the testing scenario is only reproducible by replay of the recorded data in post-processing environment, due to the changing environment in the reality, e.g. position of real satellites, ionosphere delay and troposphere delay. The post-processing environment runs on the MATLAB \& Simulink platform on a laptop with an Intel Core i7-7700HQ CPU @ 2.80GHz. 


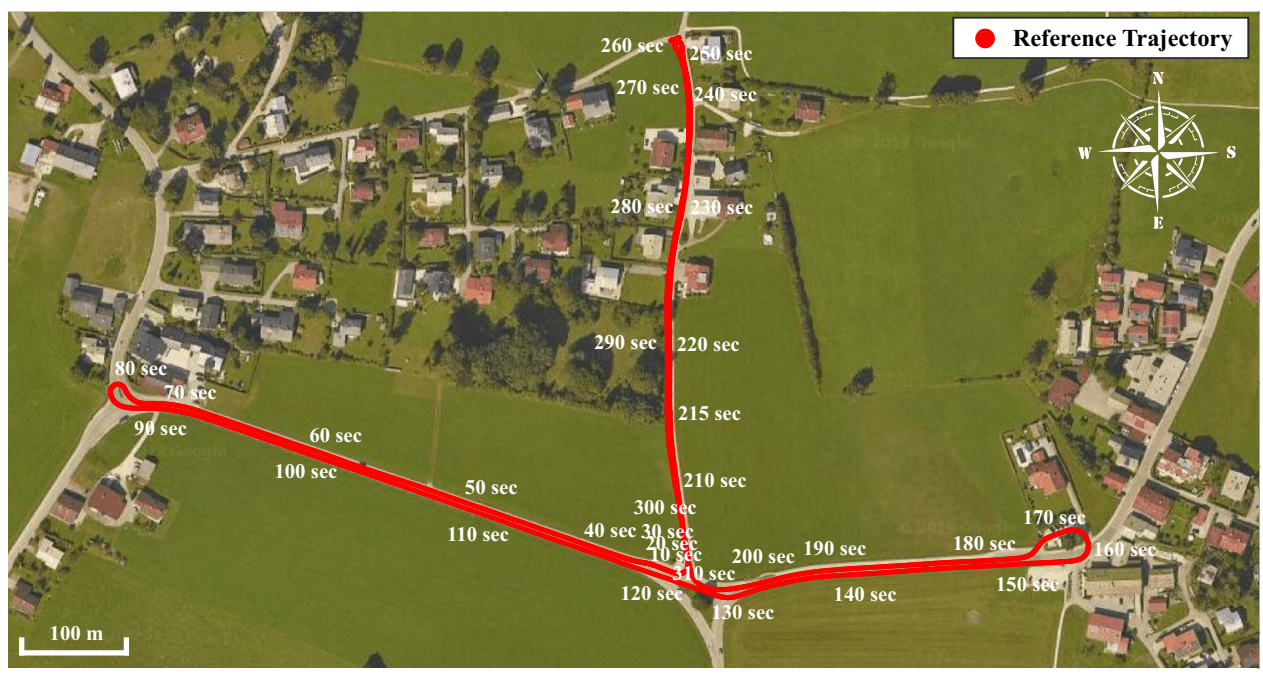

Figure 4: Reference trajectory using a RTK capable GNSS receiver, C)2019 GeoBasis-DE/BKG (C)2009), Google

During this drive, two feared events occur, which are range errors intentionally generated by GATE system. These errors are visualized in Fig. 5, which shows the pseudorange residuals of GPS and Galileo signals during this drive. The pseudorange residuals are calculated as the difference between measured and true pseudoranges. Here, true pseudoranges are estimated with the highly accurate RTK reference solution and satellite positions. Fig. 5 shows that from second 108 to 172 and from second 188 to 252, the pseudorange residuals from four Galileo satellites E10, E16, E17, E23 are extremely high. These are the two periods when feared events occur, which is verified by the experiment report from IFEN GmbH. These two periods are marked with the gray dashed lines in the following figures.

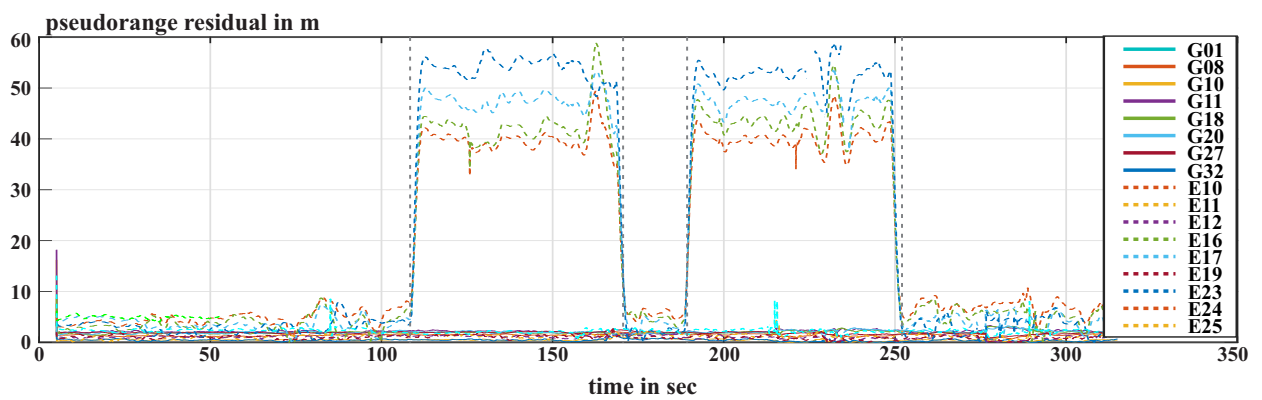

Figure 5: GNSS pseudorange residuals 


\subsection{Experimental Results}

\subsubsection{Velocity Integration without GNSS update}

In this section, dynamic propagation using velocity information is validated. The box $\left[\boldsymbol{x}^{a}\right]$ is initialized when the first GNSS measurement is available. After that, the trust box is only propagated with the velocity information without further GNSS update. Various parameter settings and potential situations are evaluated and discussed. The three settings are:

- Setting 01: The trust box is propagated only with velocity information without considering their variance, which means the velocity error bound is zero.

- Setting 02: The trust box is propagated in the same way as Setting 01. The faulty GNSS measurements from E10, E16, E17, E23 are manually excluded.

- Setting 03: The trust box is propagated with velocity information and its variance. The error bound of velocity is estimated with the approach from Sec 2.3.2 without measurement relax using the estimated variance.

Experimental results are shown in Fig. 6. The three subfigures are the difference between propagated upper and lower bounds of antenna position in NED coordinates and their reference, respectively. It can be observed that with Setting 01 the lower and upper bounds are evenly distributed around the reference, before the first feared event. During the first feared event, the propagated bounds in north direction shift downwards, which makes the distance between the upper bound and the reference smaller. During the second feared event, the bounds drift further downwards and eventually cross the reference, which makes it an invalid propagation. The reason is that the huge pseudorange error results in faulty velocity estimation of the navigation filter. This can be verified with the Setting 02. After excluding the feared event manually, the velocity propagation is valid during the whole experiment. Introducing the estimated variance of the velocity estimation solves this problem, which is verified with Setting 03. The width of the trust box increases, when no GNSS update is carried out, because the width of the trust box is the integration of velocity error bounds.

It can also be observed that the width of the trust box is no larger than 100 meters, although there is no GNSS update in 300 seconds. This means, the confidential level of the velocity information can be set higher, if periodic GNSS updates are carried out. Because GNSS updates will reduce the size of trust box periodically, this will not introduce much extra computational load.

\subsubsection{Identification Correctness and Accuracy Improvement}

In [11], a situation is described in the experimental validation chapter, that RAIM with parity space fails to identify all faults in a very short period, while RSIVIA is capable to identify all faulty pseudoranges. The comparison between RAIM and RSIVIA under such condition is given in [11], and therefore, is not repeated in the present publication. In this section, the GNSS update as well as the integration 


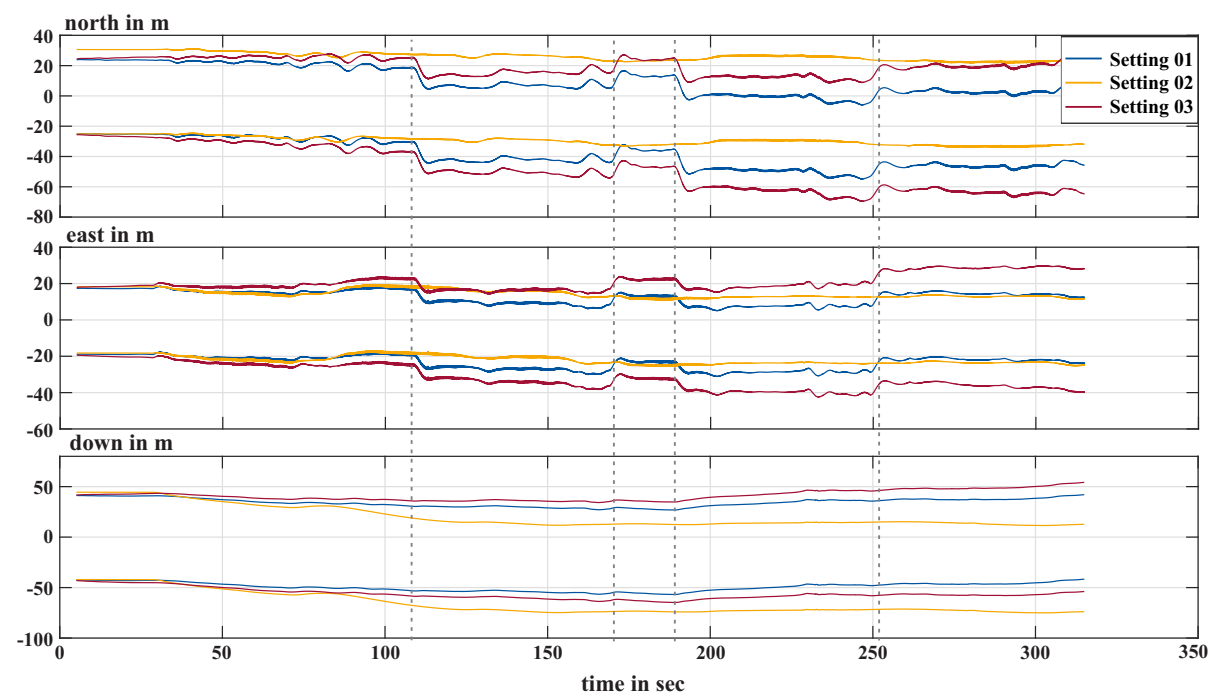

Figure 6: Validation of trust box propagation using velocity information without GNSS update: estimated upper and lower bounds minus the reference

of velocity information and RAIM are evaluated, with respect of identification correctness, the accuracy improvement and the computational load. To achieve the comparison of these, three settings are used in this section:

- Setting 04: GNSS update runs in $1 \mathrm{~Hz}$. Each RSIVIA process starts with an initial guess of an arbitrary big box.

- Setting 05: GNSS update runs in $1 \mathrm{~Hz}$. The trust box is propagated with velocity information (Sec. 2.4). RSIVIA starts with propagated trust box.

- Setting 06: The complete proposed approach in Sec. 2.5 is applied here. In case of no RAIM fault alert, the maximum duration without GNSS update $t_{u, \max }$ is 60 seconds.

Fig. 7 shows the experimental results. The first subfigure shows the fault identification result from the three settings and the satellites availability during the test drive. As already shown in Fig. 5, the pseudorange error gradually increases at the beginning of each feared event. This type of pseudorange fault is introduced in [14] as most hazardous fault model for snapshot integrity monitoring, because the state estimation are corrupted before the fault is identified.

This can be verified by Fig. 7. When the first feared even starts (at 108.4 seconds), the erroneous pseudorange is first identified with Setting 06 (at 109.87 seconds), then with Setting 04 (at 110.20 seconds) and finally with Setting 05 (at 110.67 seconds). The reason of the identification delay with Setting 04 and 05 is, that the GNSS update runs in $1 \mathrm{~Hz}$ due to the high computational load, while 


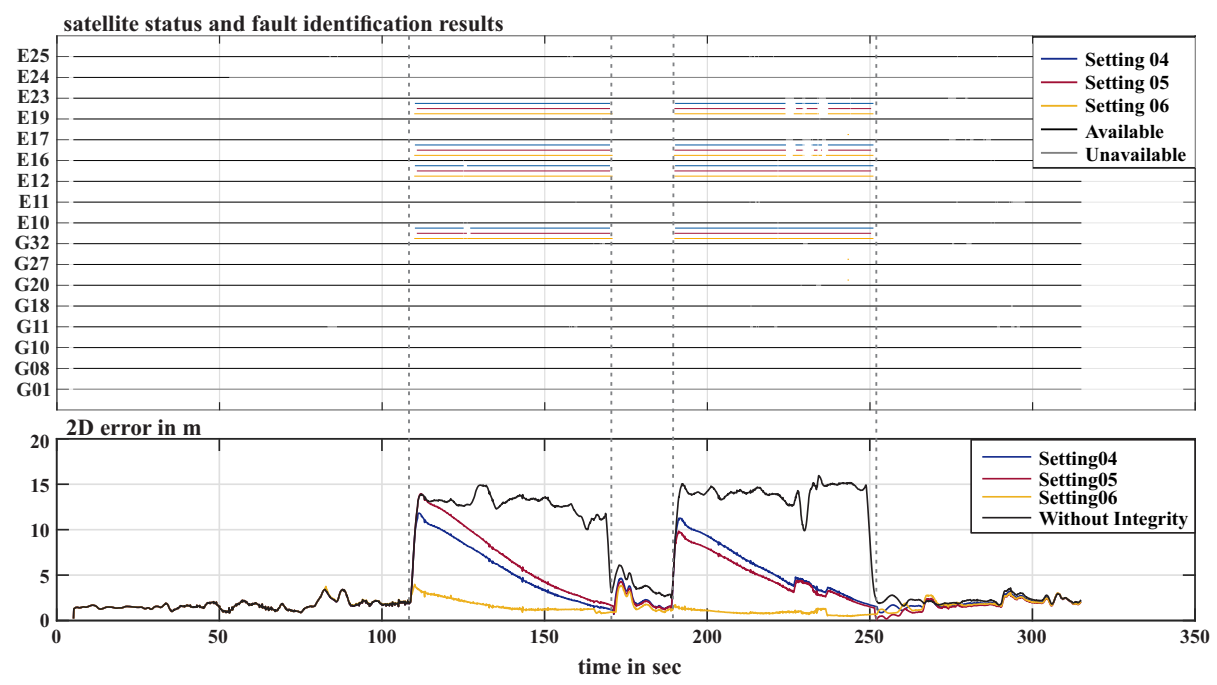

Figure 7: Validation of fault identification and accuracy improvement

the GNSS measurements are received in $10 \mathrm{~Hz}$. Furthermore, the GNSS update is triggered timely by RAIM with Setting 06. Although the time difference of first fault identification among three Settings is tiny, it results in significant difference in state estimation.

The second subfigure shows the 2D error as a measure of state estimation quality. Without FDE, the 2D error remains between 10 meters to 16 meters during the feared events. With Setting 04 and 05, the 2D error increases up to 14 meters and 12 meters, respectively, and converges slowly towards the accuracy without feared events. With Setting 06, the 2D error increases slightly to 3.7 meters, because the fault is identified earlier, and converges quickly to 1 meter.

Finally, the computation time is evaluated. The post-processing takes 81.70 seconds without integrity monitoring, which is the baseline of computation time. Further, the post-processing takes 276.68, 171.51 and 121.89 seconds with Setting 04, 05 and 06, respectively. Considering that GNSS update runs at $1 \mathrm{~Hz}$ both with Setting 04 and 05, the number of GNSS updates is the same. By introducing the velocity information to reduce the size of the initial guess, the average computation time of each GNSS update is reduced by $53.94 \%$, which is very important for the future real-time implementation. In contrast, RAIM reduces the total computation time by reducing the number of GNSS updates, instead of reducing the average computation time. Therefore, RAIM may not improve the real-time computational performance very much. However, introducing RAIM provides a more timely fault identification, which improves the accuracy of navigation solution. 


\section{Conclusion}

This publication presented the development of an integrity system as an extension of a tightly-coupled navigation filter within the joint-project GALILEOnautic 2. The main purpose of the integrity system is FDE of multi-faults in pseudorange measurements, such that a set of fault-free GNSS measurements can be fed into the navigation filter. In this work, a RSIVIA based FDE strategy is proposed, which involves RAIM with parity space and velocity estimation from the navigation filter. With respect to the experimental evaluation, an offline post-processing using data from GATE Berchtesgaden is carried out. In this experiment, multiple feared events are intentionally generated, which are correctly identified by the proposed approach. The measurement campaign evaluations visualize that this approach improves notably both the accuracy and robustness of the navigation filter and reduces significantly the computational load compared to the traditional RSIVIA.

In future works, a real time implementation of the designed approach is aimed. On this basis, an integrity monitoring system for all sensors integrated into the navigation system will be developed, which considers IMU, DVL and GNSS measurements. FDE will be performed on all measurements, which are used by the navigation filter, to enhance the navigation filter reliability.

\section{References}

11 Brown, R.G. A baseline GPS RAIM scheme and a note on the equivalence of three RAIM methods. Journal of The Insititute of Navigation, 39(3):301-316, 1992. DOI: $10.1002 / j .2161-4296.1992 . t b 02278 . x$.

[2] Dbouk, H. and Schön, S. Comparison of different bounding methods for providing GPS integrity information. In 2018 IEEE/ION Position Location and Navigation Symposium (PLANS), pages 355-366, Piscataway, NJ, 2018. IEEE. DOI: $10.1109 /$ PLANS . 2018.8373401.

[3] Drevelle, V. and Bonnifait, P. High integrity GNSS location zone characterization using interval analysis. Proceedings of the 22nd International Technical Meeting of the Satellite Division of The Institute of Navigation (ION GNSS 2009), pages 2178-2187, 2009.

[4] Drevelle, V. and Bonnifait, P. A set-membership approach for high integrity height-aided satellite positioning. GPS Solutions, 15(4):357-368, 2011. DOI: 10.1007/s10291-010-0195-3.

[5] European Global Navigation Satellite System Agency. GSA GNSS Market Report 2019. Publications Office of the European Union, 2019.

[6] Gehrt, J.-J., Zweigel, R., Konrad, T., and Abel, D. DVL-aided navigation filter for maritime applications. 11th IFAC Conference on Control Applications in Marine Systems, Robotics and Vehicles (IFAC CAMS 2018), pages 418-423, 2018. DOI: $10.1016 /$ j.ifacol.2018.09.451. 
[7] Jaulin, L., Kieffer, M., Didrit, O., and Walter, E. Applied Interval Analysis. Springer, 2001. DOI: $10.1007 / 978-1-4471-0249-6$.

[8] Jaulin, L., Kieffer, M., Walter, E., and Meizel, D. Guaranteed robust nonlinear estimation with application to robot localization. IEEE Transactions on Systems, Man and Cybernetics, Part C (Applications and Reviews), 32(4):374381, 2002. DOI: 10.1109/TSMCC. 2002.806747.

[9] Konrad, T., Breuer, M., Engelhardt, T., and Abel, D. State estimation for a multirotor using tight-coupling of GNSS and inertial navigation. IFACPapersOnLine, 50(1):11683-11688, 2017. DOI: 10.1016/j.ifacol.2017.08. 1684.

[10] Liu, S., Gehrt, J.-J., Abel, D., and Zweigel, R. Dual-constellation aided high integrity and high accuracy navigation filter for maritime applications. Proceeding of the 2019 International Technical Meeting of The Institute of Navigation (ION ITM), pages 762-774, 2019. DOI: 10.33012/2019.16723.

[11] Liu, S., Gehrt, J.-J., Abel, D., and Zweigel, R. Integrity of dual-constellation aided navigation filter in safety-critical maritime applications. European journal of navigation, 19(3):10-17, 2019.

[12] Pervan, B., Lawrence, D., Cohen, C., and Parkinson, B. Parity space methods for autonomous fault detection and exclusion using GPS carrier phase. Position Location and Navigation Symposium, pages 649-656, 1996. DOI: 10.1109/ PLANS.1996.509141.

[13] Rohou, S., Jaulin, L., Mihaylova, L., Le Bars, F., and Veres, S.M. Guaranteed computation of robot trajectories. Robotics and Autonomous Systems, 93:7684, 2017. DOI: $10.1016 / j$.robot .2017 .03 .020 .

[14] Tanil, C., Khanafseh, S., Joerger, M., and Pervan, B. Sequential integrity monitoring for kalman filter innovations-based detectors. In Proceedings of the 31st International Technical Meeting of The Satellite Division of the Institute of Navigation (ION GNSS+ 2018), pages 2440-2455. Institute of Navigation, 2018. DOI: $10.33012 / 2018.15975$. 